\title{
Resveratrol enhances vascular reactivity in mice following lipopolysaccharide challenge via the RhoA-ROCK-MLCP pathway
}

\author{
XU-QING WANG, YU-PING ZHANG, LI-MIN ZHANG, NIU-NIU FENG, \\ MING-ZHU ZHANG, ZI-GANG ZHAO and CHUN-YU NIU \\ Institute of Microcirculation, Hebei North University, Zhangjiakou, Hebei 075000, P.R. China
}

Received December 2, 2015; Accepted March 31, 2017

DOI: $10.3892 / \mathrm{etm} .2017 .4486$

\begin{abstract}
The aim of the present study was to identify whether sepsis-induced vascular hyporeactivity is associated with microcirculation disturbance and multiple organ injuries. The current study assessed the impact of resveratrol (Res) treatment on lipopolysaccharide (LPS) challenge mediated vascular hyporeactivity. Effects of Res treatment (30 mg/kg; i.m.) at $1 \mathrm{~h}$ following LPS stimulation $(5 \mathrm{mg} / \mathrm{kg}$; i.v.) on the survival time, mean arterial pressure (MAP), and maximal difference of $\operatorname{MAP}(\triangle \mathrm{MAP})$ to norepinephrine (NE; $4.2 \mu \mathrm{g} / \mathrm{kg}$ ) in mice were observed. The reactivity to gradient $\mathrm{NE}$ of isolated mesenteric arterioles and the association with the RhoA-RhoA kinase (ROCK)-myosin light chain phosphatase (MLCP) pathway were investigated by myography, and the signaling molecule protein levels were assessed using ELISA. Res treatment prolonged the survival time of mice subjected to LPS challenge, but did not prevent the LPS-induced hypotension and increase in $\triangle$ MAP. Res treatment and RhoA agonist U-46619 incubation prevented LPS-induced vascular hyporeactivity ex vivo, which were suppressed by incubation with ROCK inhibitor Y-27632. LPS-induced vascular hyporeactivity was not affected by the MLCP inhibitor okadaic acid incubation, but was further downregulated by the co-incubation of OA plus Y-27632. The inhibiting effect of Y-27632 on Res treatment was eradicated by incubation with U-46619. Furthermore, RhoA inhibitor C3 transferase did not significantly inhibit the enhancing role of Res treatment, which was further increased by U-46619 plus C3 transferase co-incubation. In addition, Res treatment eradicated the LPS-induced decreases in $\mathrm{p}-\mathrm{RhoA}$ and p-Mypt1 levels and increases in MLCP levels. The results
\end{abstract}

Correspondence to: Professor Chun-Yu Niu or Professor Zi-Gang Zhao, Institute of Microcirculation, Hebei North University, 11 Diamond South Road, Zhangjiakou, Hebei 075000, P.R. China E-mail: lymphatics@126.com

E-mail: zzghyl@126.com

Key words: resveratrol, lipopolysaccharide, vascular reactivity, RhoA-ROCK-MLCP, intravenous injection of the present study indicate that post-treatment of Res significantly ameliorates LPS-induced vascular hyporeactivity, which is associated with the activation of the RhoA-ROCK-MLCP pathway.

\section{Introduction}

A variety of serious infection factors induce endotoxic and septic shock, which are common clinical pathological processes $(1,2)$. A previous study has demonstrated that intravenous injection of lipopolysaccharide (LPS) attenuated both norepinephrine (NE)-induced vascular contraction and acetylcholine-induced vasodilation in the aortas of rats (3). Liang et al (4) also indicated that LPS treatment decreased the vascular reactivity of superior mesenteric arteries to a $\alpha 1 \mathrm{AR}$ agonist (phenylephrine) after $2 \mathrm{~h}$ in rabbits. These results demonstrated that endotoxic shock induced hypovascular reactivity during the late stage, which is an important factor of microcirculation disturbance and tissue hypoperfusion, and further leads to multiple organ injuries and dysfunction $(1,2)$. A number of studies have indicated that resveratrol (Res) has a variety of biological activities $(5,6)$. Previous studies have also revealed that Res alleviated the endotoxic shock-induced structural damage and dysfunction of vital organs, including the lung, kidney and liver (7-9). Mechanisms of Res have been demonstrated to be associated with reducing oxidative stress and inflammation and improving microcirculation perfusion status (8-13). However, whether or not Res enhances vascular reactivity following endotoxic shock remains unclear. Tseng et al (14) indicated that LPS-induced hyporeactivity of isolated mesenteric arteries is associated with the decreased phosphorylation of Rho kinase (ROCK) and suppression of Ras homolog gene family, member A (RhoA) activities in smooth muscle of arteries. However, an increase of RhoA activity may compensate for vascular reactivity in early endotoxaemia induced by LPS injection, and suppression of RhoA activity may eventually lead to vascular hyporeactivity in late endotoxaemia (15). In conclusion, the RhoA has an important role in LPS-induced vascular hyporeactivity. Therefore, the present study hypothesized that Res treatment alleviates LPS-induced vascular hyporeactivity via the activation of the RhoA-ROCK-myosin light chain phosphatase (MLCP) 
pathway. To assess this hypothesis, the current study observed the role and mechanism of Res on vascular reactivity subjected to LPS challenge using a microvascular tension measurement technique. The current study may provide novel experimental evidence for the treatment of vascular hyporeactivity induced by endotoxic shock.

\section{Materials and methods}

Animals. A total of 72 healthy, male and specific pathogen-free BALB/c mice (weight, 23-27 g; age, 3 months) were purchased from the National Institutes for Food and Drug Control [Beijing, China; SCXK (jing) 2009-0017], and housed in clear plastic cages at a temperature of $23 \pm 1^{\circ} \mathrm{C}$ and humidity of $40-50 \%$, with a 12 -h light/dark cycle and access to water and food as indicated. Mice were randomly divided into four groups, as follows: Sham, Sham + Res, LPS and LPS + Res ( $n=18$ per group). A total of 6 mice in each group were used for the observation of survival time, 6 mice in each group were used for the investigation of pressor response to $\mathrm{NE}$ in vivo and the remaining 6 mice in each group were used for the investigation of vascular reactivity ex vivo. Prior to the experiment, the mice were fasted for $12 \mathrm{~h}$ and were allowed ad libitum access to water. All experimental procedures involving animals were reviewed and approved by the Institutional Animal Care and Use Committee of Hebei North University (Zhangjiakou, China) and conformed to the National Institutes of Health guidelines. All efforts were made to minimize the suffering of the animals during the experiment.

Model preparation and survival assessment. Mice were anesthetized with $1 \%$ pentobarbital sodium $(70 \mathrm{mg} / \mathrm{kg}$; Merck KGaA, Darmstadt, Germany; H8060) in a volume of 0.16-0.19 ml. Under the guidance of a stereomicroscope (SZ61; Olympus Corporation, Shanghai, China), surgery on the neck was performed in order to separate the right jugular vein from the surrounding tissues. Following a 30 -min stabilization period, LPS administration $(0.5 \%$, $5 \mathrm{mg} / \mathrm{kg}$ Escherichia coli; O111:B4; Sigma-Aldrich; Merck $\mathrm{KGaA}$ ) was performed via the right jugular vein in the LPS and LPS + Res groups. The same amount of saline $(\mathrm{NaCl})$, instead of LPS, was administered in the sham and sham + Res groups. Subsequently, jugular vein ligation, wound suture and disinfection with iodine were performed in sequence. After $1 \mathrm{~h}$ of LPS or saline administration, an intramuscular injection of Res (30 mg/kg, Sigma-Aldrich; Merck KGaA) was administered to mice in the LPS + Res and sham + Res groups. The dosage of Res used in the present study was in accordance with previous studies $(16,17)$. The vehicle, dimethyl sulfoxide, was administered instead of Res to the mice in the sham and LPS groups. Subsequently, the survival assessment was performed. Because the mice in the LPS and LPS + Res groups were all sacrificed within $24 \mathrm{~h}$, therefore, in this current study, survival time and survival rate of $24 \mathrm{~h}$ were recorded in each group $(n=6)$ and the observed end-point was $24 \mathrm{~h}$. Surviving mice in the sham and sham + Res groups were anesthetized at $24 \mathrm{~h}$ with $1 \%$ pentobarbital sodium $(70 \mathrm{mg} / \mathrm{kg})$ via intraperitoneal injection and sacrificed using cervical dislocation. The full duration of this experiment was $24 \mathrm{~h}$, while subsequent experiments were completed.
Pressor response to NE in vivo. In addition to the aforementioned treatment, following anesthetization and administration of intravenous heparin $(500 \mathrm{U} / \mathrm{kg}, 1 \mathrm{ml} / \mathrm{kg}$; RongSheng Pharmaceutical Co., Ltd., Jiaozuo, China), the mice were subjected to femoral surgery in order to separate the right femoral artery from the surrounding tissues. A minimally heparinized microcatheter was subsequently introduced into the right femoral artery to monitor mean arterial pressure (MAP) during the experiment, using the PowerLab biological signal collection and processing system (ML818; ADInstruments Australia, Bella Vista, NSW, Australia). At $6 \mathrm{~h}$ after LPS or saline administration, NE $(4.2 \mu \mathrm{g} / \mathrm{kg})$ was injected into the jugular vein. Changes in MAP at pre- and post-administration of NE were recorded and the maximal difference of MAP ( $\triangle \mathrm{MAP})$ was presented as an index of vascular reactivity in vivo $(18,19)(\mathrm{n}=6$ per group). Subsequently, the mice were sacrificed by cervical dislocation. The present experiment continued $7 \mathrm{~h}$ from anesthetization to sacrifice of mice, and there was no animal sacrificed during the experiment.

Vascular reactivity ex vivo. A total of 24 mice received treatment ( $n=6$ from each group) at the same observed end-point as described. At $6 \mathrm{~h}$ after LPS or corresponding sham administration, under anesthesia as described above, a laparotomy was performed prior to cervical dislocation and a loop of intestine with mesenteric tissues was removed and placed into chilled physiological saline solution (PSS; in mmol/l: $118.99 \mathrm{NaCl}$, $4.69 \mathrm{KCl}, 1.17 \mathrm{MgSO}_{4}, 25.0 \mathrm{NaHCO}_{3}, 1.20 \mathrm{KH}_{2} \mathrm{PO}_{4}, 2.50 \mathrm{CaCl}_{2}$, 0.03 EDTA $\mathrm{Na}_{2}$ and 5.5 glucose at $\mathrm{pH}$ 7.3-7.4), which was continuously bubbled with $95 \% \mathrm{O}_{2} / 5 \% \mathrm{CO}_{2}$. Suitable mesentery arteries were identified and excised under the operating microscope. Four segments of $2 \mathrm{~mm}$ in length were prepared and transferred to an isolated vessel chamber of wire myograph system (620M; Danish Myo Technology A/S, Aarhus N, Denmark) filled with chilled PSS. Each vascular ring was cannulated onto two $2.5-\mathrm{cm}$ lengths of stainless steel wire (diameter, $40 \mu \mathrm{m}$ ) in the raw; namely, no traction and tension. Chambers were mounted onto a wire myograph. Following a 5-min stabilization period, the passive tension (preload) calculated with the standardized program was initially set using the myograph system. Normalization was performed by stretching the segment stepwise and measuring the micrometer and force readings. These data are converted into values of internal circumference $(\mu \mathrm{m})$ and wall tension $\mathrm{T}(\mathrm{mN} / \mathrm{mm})$, respectively. Subsequently, the bath temperature was slowly increased to $37^{\circ} \mathrm{C}$ within $20 \mathrm{~min}$ and the vessel was equilibrated for $30 \mathrm{~min}$ in PSS, which was continuously bubbled with $95 \% \mathrm{O}_{2} / 5 \% \mathrm{CO}_{2}$, with the solution being changed and the transducer re-zeroed at the end of the equilibration period. When the temperature reached $37^{\circ} \mathrm{C}$, the vessel underwent normalization and activation. The vessel was activated with $\mathrm{NE}\left(1 \times 10^{-3} \mathrm{~mol} / \mathrm{l}\right)$ and a high potassium solution $\left(2.70 \mathrm{NaCl}, 120.0 \mathrm{KCl}, 0.45 \mathrm{MgSO}_{4}\right.$, $25.0 \mathrm{NaHCO}_{3}, 1.20 \mathrm{KH}_{2} \mathrm{PO}_{4}, 2.50 \mathrm{CaCl}_{2}$ and 5.5 glucose $\mathrm{mmol} / \mathrm{l}$ at $\mathrm{pH}$ 7.3-7.4). Following 3 to 5 washes with PSS solution for $10 \mathrm{~min}, \mathrm{NE}$ was administered to all samples at final concentrations of $1 \times 10^{-7}, 3 \times 10^{-7}, 1 \times 10^{-6}, 3 \times 10^{-6}, 1 \times 10^{-5}$ and $3 \times 10^{-5} \mathrm{~mol} / 1$ using a concentration accumulation method with an interval of $80 \mathrm{sec}$, respectively, as in previous studies $(18,19)$. The difference of tension and baseline induced by $\mathrm{NE}$ was recorded using the PowerLab system as the vascular tension response. Following 
the experiment, the dose-response line was constructed using SigmaPlot software, version 11.0 (Systat Software, Inc., San Jose, CA, USA). Effective concentration at 50\% $\left(\mathrm{EC}_{50}\right)$ and negative $\log$ of molar concentration $\left(\mathrm{pD}_{2}\right)$ were used to identify the - $\log 50 \%$ effective concentration of the dose-response line using the curve-fitting method. Dose-response line, maximum tension (Emax), and $\mathrm{pD}_{2}$ were presented as indices of vascular tension reactivity. The full duration of micro-vascular reactivity observation was $3 \mathrm{~h}$.

When the contractile response to NE was measured in PSS, the vessels were incubated with tool reagents of RhoA, ROCK and MLCP in an isolated vessel chamber with PSS bubbled with $95 \% \mathrm{O}_{2} / 5 \% \mathrm{CO}_{2}$ for $10 \mathrm{~min}$, and then the contractile response to $\mathrm{NE}$ was measured again to determine the role of these signal molecules in vascular reactivity. Briefly, two segments of microvascular vessels from the sham group were treated with RhoA agonist U-46619 (Alexis, Inc., Lausen, Switzerland; final concentration, $5 \times 10^{-7} \mathrm{~mol} / \mathrm{l}$ ) or MLCP inhibitor okadaic acid (OA; Enzo, Inc., New York, NY, USA; final concentration, $1 \times 10^{-6} \mathrm{~mol} / \mathrm{l}$ ) for $10 \mathrm{~min}$ to measure the contractile response to NE. Following 3 to 5 washes with PSS, the two segment vessels were treated with RhoA inhibitor C3 transferase (Cytoskeleton, Inc., Denver, CO, USA; final concentration, $60 \mu \mathrm{g} / \mathrm{l}$ ) or ROCK inhibitor Y-27632 (Alexis, Inc.; final concentration, $6 \times 10^{-6} \mathrm{~mol} / \mathrm{l}$ ) for $10 \mathrm{~min}$ for the measurement of the response to NE. Vessels from the sham + Res group were treated with the same procedure as the sham group. Only one segment of microvascular vessel from the LPS group was treated with OA and measured for a response to NE, after 3 to 5 washes with PSS. The segment was then treated with OA and Y-27632 together and the response to NE was measured. The other segment of microvascular vessel from the LPS group was treated with U-46619 and U-46619 plus Y-27632 for the measurement of the response to NE. Similarly, the vascular reactivity to NE of the LPS + Res group was measured following treatment with Y-27632, Y-27632 plus U-46619, C3 transferase and C3 transferase plus U-46619 for the measurement of the response to NE.

Measurement of p-RhoA, p-Mypt1 and MLCP. At $6 \mathrm{~h}$ after LPS or corresponding treatment, the mesenteric arteries were separated under an operating microscope, and stored at -75 to $-80^{\circ} \mathrm{C}$. Subsequently, the vascular tissue was pulverized using a MagNA Lyser automatic tissue homogenizer (Roche Diagnostics International AG, Rotkreuz, Switzerland) with $50 \mu 1$ cell lysis buffer (Beyotime Biotechnology Research Institute, Shanghai, China), and was centrifuged at $850 \mathrm{x} \mathrm{g}$ at $0-4^{\circ} \mathrm{C}$ for $10 \mathrm{~min}$ using the $3-30 \mathrm{~K}$ high-speed refrigerated Laborzentrifugen (Sigma Laborzentrifugen $\mathrm{GmbH}$, Osterode am Harz, Germany). Phosphorylated (p-) RhoA, p-myosin phosphatase target subunit 1 (Mypt1) and MLCP levels were examined using the mouse-specific ELISA kits (Jiangsu Hope, Inc., Zhenjiang, China; for p-RhoA, HBT-0587M; p-Mypt1, HBT-0684M; and MLCP, HBT-0622M) in accordance with the manufacturer's protocol. Antibodies for p-RhoA (ab41435; 1:2,000), p-Mypt1 (ab59203; 1:4,000), and MLCP (ab59235; 1:4,000) were purchased from Abcam (Cambridge, MA), and used for the ELISA analysis. Protein concentration of the vascular homogenate was measured using a bicinchoninic acid protein assay kit

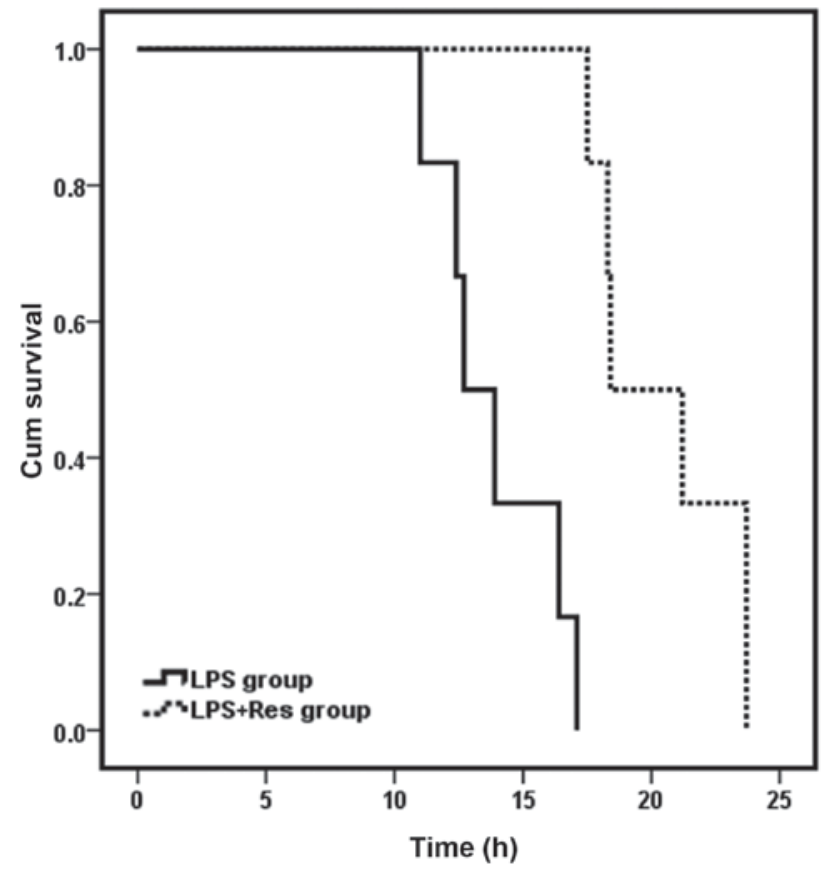

Figure 1. Res treatment prolonged the survival time of mice subjected to LPS challenge through intravenous injection. Res, resveratrol; LPS, lipopolysaccharide.

(Applygen Technologies Inc., Beijing, China), according to the manufacturer's instructions, for the normalization of protein levels.

Statistical analysis. Data in this study were expressed as mean + or \pm standard deviation or mean \pm standard error as indicated. For the comparison of more than two conditions, a one-way analysis of variance (ANOVA) with Turkey's post hoc test or repeated-measures ANOVA with Bonferroni post hoc test were used. For survival time, Kaplan-Meier analysis was used, followed by a Log-rank test. $\mathrm{P}<0.05$ was considered to indicate a statistically significant difference.

\section{Results}

Effect of Res treatment on the survival time of mice subjected to LPS challenge. Observation of survival time was initiated at the point of intravenous injection of $\mathrm{NaCl} / \mathrm{LPS}$. Mice in the sham and sham + Res groups survived for $24 \mathrm{~h}$ and the $24 \mathrm{~h}$ survival rate was $100 \%$ for these groups. Survival time of mice in the LPS + Res group was $20.45 \pm 2.81 \mathrm{~h}$, which was significantly longer than the LPS group $(13.89 \pm 2.37 \mathrm{~h} ; \mathrm{P}<0.05)$. However, the 24-h survival rate for the LPS and LPS + Res groups was $0 \%$. The survival curve of the LPS and LPS + Res groups is presented in Fig. 1.

Effect of Res treatment on MPA and $\triangle M A P$ levels of mice subjected to LPS challenge. In the LPS and LPS + Res groups, MAP was significantly decreased and $\triangle \mathrm{MAP}$ was significantly increased when compared with that of the sham and sham + Res groups, $6 \mathrm{~h}$ after intravenous injection of $\mathrm{NaCl} / \mathrm{LPS}$ $(\mathrm{P}<0.05$; Fig. 2). There were no significant differences in the MAP and $\triangle$ MAP levels between the sham and sham + Res groups or the LPS and LPS + Res groups ( $\mathrm{P}>0.05$; Fig. 2). 
A

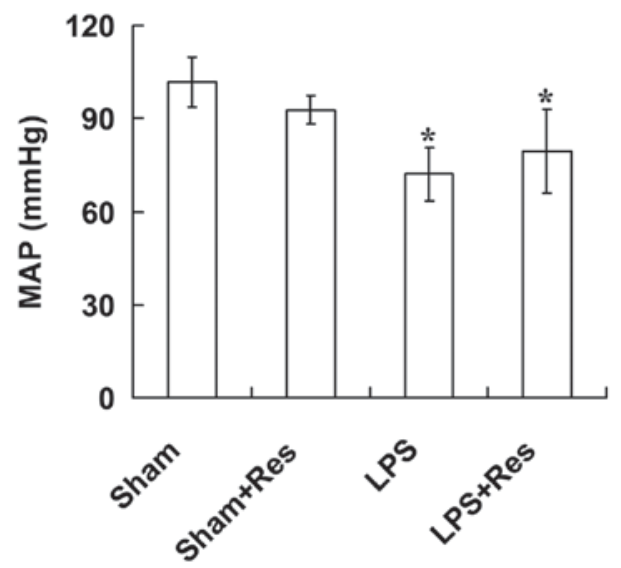

B

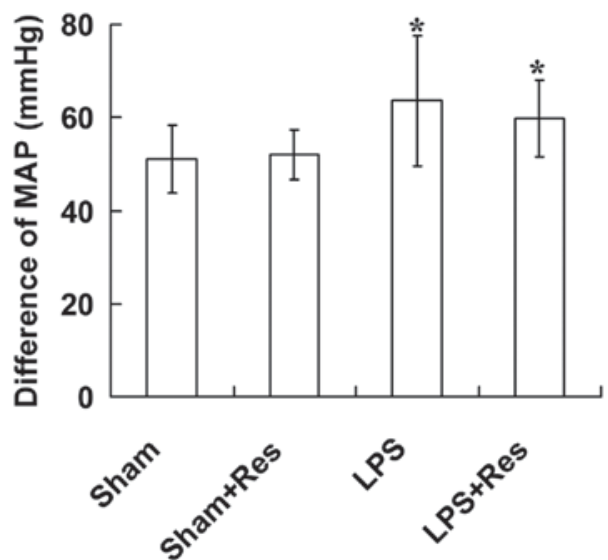

Figure 2. (A) Changes in MAP and (B) difference of MAP following the administration of norepinephrine, $6 \mathrm{~h}$ after the LPS intravenous challenge in mice. ${ }^{*} \mathrm{P}<0.05$ vs. sham and sham + Res groups. Data are presented as mean \pm standard deviation, $n=6$. MAP, mean artery pressure; LPS, lipopolysaccharide; Res, resveratrol.

A a

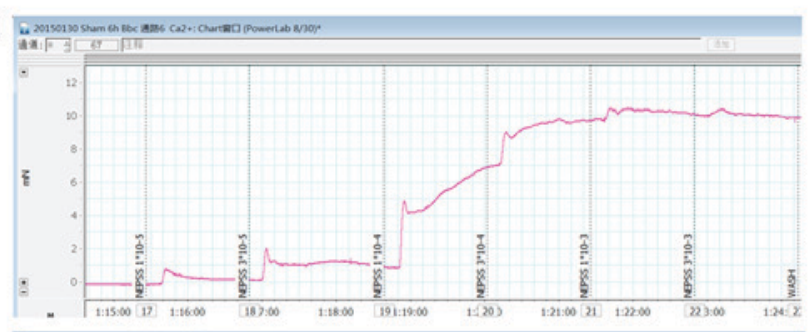

C

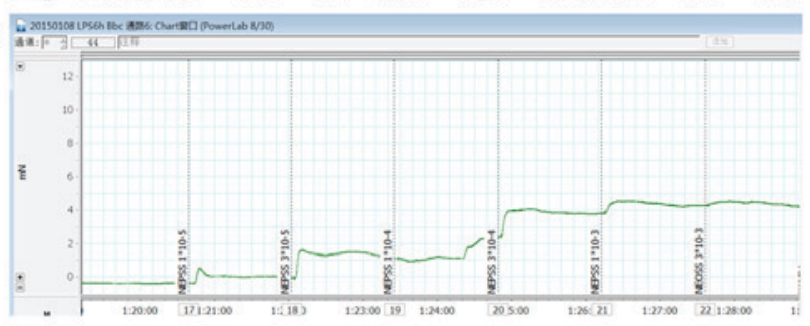

b
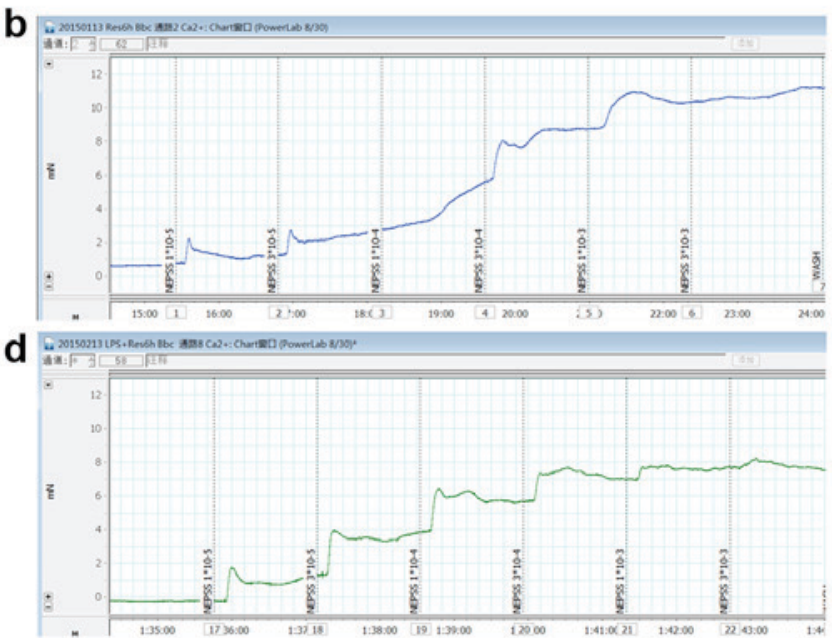

B

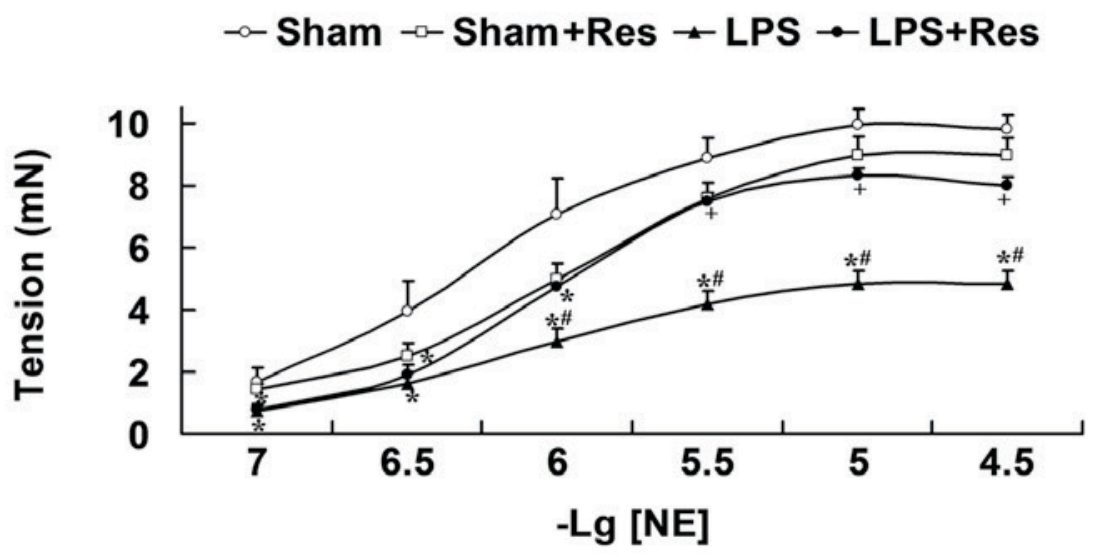

Figure 3. Res treatment enhanced the micro-vascular reactivity to NE in mice following LPS intravenous injection. (A) Images of the original recording for (A-a) sham, (A-b) sham + Res, (A-c) LPS and (A-d) LPS + Res groups. (B) Results demonstrating the concentration-response curves of arteriole rings following treatment with $N E$ to assess the tension. ${ }^{*} \mathrm{P}<0.05$ vs. sham group; ${ }^{\prime \prime} \mathrm{P}<0.05$ vs. sham + Res group; ${ }^{+} \mathrm{P}<0.05$ vs. LPS group. Data are presented as mean \pm standard error, $\mathrm{n}=6$. Res, resveratrol; NE, norepinephrine; LPS, lipopolysaccharide.

Effect of Res treatment on the vascular reactivity of mice subjected to LPS challenge. Fig. 3 and Table I indicate that there were no significant differences in the response of mouse arteriole rings to NE in the sham and sham + Res groups
( $\mathrm{P}>0.05$ ), with the exception of the $1 \times 10^{-6} \mathrm{~mol} / \mathrm{l}$ concentration between the sham and sham + Res groups. The response of mouse arteriole rings to NE in the LPS group was significantly decreased when compared with that of the sham group 
Table I. Effects of Res on the Emax and $\mathrm{pD}_{2}$ of micro-vessels to norepinephrine in mice following LPS intravenous injection.

\begin{tabular}{lcc}
\hline Group & Emax, $\mathrm{mN}$ & $\mathrm{pD}_{2}$ \\
\hline Sham & $10.03 \pm 1.16$ & $4.20 \pm 0.28$ \\
Sham + Res & $9.12 \pm 1.41$ & $3.95 \pm 0.14^{\mathrm{a}}$ \\
LPS & $4.89 \pm 1.10^{\mathrm{a}, \mathrm{b}}$ & $4.05 \pm 0.19$ \\
LPS + Res & $8.30 \pm 0.59^{\mathrm{a}, \mathrm{c}}$ & $4.04 \pm 0.08$ \\
\hline
\end{tabular}

${ }^{\mathrm{a}} \mathrm{P}<0.05$ vs. sham group; ${ }^{\mathrm{b}} \mathrm{P}<0.05$ vs. sham + Res group; ${ }^{\mathrm{c}} \mathrm{P}<0.05$ vs. LPS group. Data are presented as mean \pm standard error $(n=6)$. Res, resveratrol; Emax, maximum tension; $\mathrm{pD}_{2}$, negative log of molar concentration; LPS, lipopolysaccharide.

for $3 \times 10^{-5}, 1 \times 10^{-5}, 3 \times 10^{-6}, 1 \times 10^{-6}, 3 \times 10^{-7}$ and $1 \times 10^{-7} \mathrm{~mol} / 1$, and the sham + Res group for $3 \times 10^{-5}, 1 \times 10^{-5}, 3 \times 10^{-6}$ and $10^{-6} \mathrm{~mol} / 1$ $(\mathrm{P}<0.05)$. Emax of the LPS group was significantly decreased compared with that of the sham and sham + Res groups $(\mathrm{P}<0.05)$. Concentration-response curves of arteriole rings to NE in the LPS + Res group were shifted markedly to the left, the tension reactivity induced by $\mathrm{NE}$ at $3 \times 10^{-5}, 1 \times 10^{-5}$ and $3 \times 10^{-6} \mathrm{~mol} / \mathrm{l}$ and the Emax were significantly enhanced compared with that of the LPS group $(\mathrm{P}<0.05)$.

Role of the RhoA-ROCK-MLCP signal pathway in Res treatment improved the vascular reactivity in vitro of mice subjected to LPS challenge. Results from different tool agents on the arteriole rings reactivity to NE in the sham mice indicated that the RhoA agonist U-46619 increased the response of arteriole rings (at $3 \times 10^{-7}$ and $1 \times 10^{-7} \mathrm{~mol} / 1$ of $\mathrm{NE}$ ), but exhibited no significant difference in Emax, and that both the RhoA inhibitor C3 transferase (at $3 \times 10^{-5}$ and $1 \times 10^{-5} \mathrm{~mol} / 1$ of NE) and ROCK inhibitor Y-27632 (at $3 \times 10^{-5}, 1 \times 10^{-5}, 3 \times 10^{-6}$, $1 \times 10^{-6}$ and $3 \times 10^{-7} \mathrm{~mol} / 1$ of $\mathrm{NE}$ ) significantly decreased the response $(\mathrm{P}<0.05$; Fig. 4A; Table II). Emax and Y-26732 also significantly decreased the response of $\mathrm{PD}_{2}$ of arteriole rings $(\mathrm{P}<0.05$; Fig. 4A; Table II). However, the MLCP inhibitor OA had no significant effect $(\mathrm{P}>0.05)$. Similarly, U-46619 treatment increased the response (at $3 \times 10^{-7}$ and $1 \times 10^{-7} \mathrm{~mol} / \mathrm{l}$ of NE) and Y-27632 incubation significantly decreased the response (at $3 \times 10^{-5}, 1 \times 10^{-5}, 3 \times 10^{-6}, 1 \times 10^{-6}$ and $3 \times 10^{-7} \mathrm{~mol} / 1$ of $\mathrm{NE}$ ) of Emax and the $\mathrm{PD}_{2}$ of arteriole rings compared with the sham+Res group $(\mathrm{P}<0.05$; Fig. 4B; Table II).

As presented in Fig. 4C and Table II, RhoA agonist U-46619 increased the response of arteriole rings harvested from the LPS challenged mice (at $1 \times 10^{-5}, 3 \times 10^{-6}, 1 \times 10^{-6}, 3 \times 10^{-7}$, and $1 \times 10^{-7} \mathrm{~mol} / 1$ of $\left.\mathrm{NE} ; \mathrm{P}<0.05\right)$, which was significantly reduced following ROCK inhibitor Y-27632 co-incubation (at $3 \times 10^{-5}$, $1 \times 10^{-5}, 3 \times 10^{-6}, 1 \times 10^{-6}, 3 \times 10^{-7}$ and $1 \times 10^{-7} \mathrm{~mol} / 1$ of $\mathrm{NE}$; $\left.\mathrm{P}<0.05\right)$, along with decreased Emax. The MLCP inhibitor, OA, had no significant effect on arteriole rings from LPS-challenged mice. However, the combination of OA and Y-27632 significantly decreased the response to NE (at $3 \times 10^{-5}, 1 \times 10^{-5}, 3 \times 10^{-6}$, $1 \times 10^{-6}$ and $3 \times 10^{-7} \mathrm{~mol} / \mathrm{l}$ ), Emax and $\mathrm{PD}_{2}$ of the arteriole rings harvested from LPS challenged mice and, following treatment with $\mathrm{OA}(\mathrm{P}<0.05$; Fig. 4C).

As indicated in Fig. 4D and Table II, ROCK inhibitor Y-27632 significantly decreased the response to NE $\left(3 \times 10^{-5}\right.$,
Table II. Effects of agonists or inhibitors of RhoA-RhoA kinase-myosin light chain phosphatase pathway on the Emax and $\mathrm{pD}_{2}$ of micro-vessels to norepinephrine.

\begin{tabular}{lcc}
\hline Group & Emax, $\mathrm{mN}$ & $\mathrm{pD}_{2}$ \\
\hline Sham & $9.73 \pm 1.14$ & $4.16 \pm 0.32$ \\
Sham + U-46619 & $9.55 \pm 0.78$ & $3.96 \pm 0.41$ \\
Sham + C3 & $7.78 \pm 0.86^{\mathrm{a}}$ & $4.15 \pm 0.27$ \\
Sham & $10.21 \pm 1.28$ & $4.25 \pm 0.30$ \\
Sham + OA & $9.50 \pm 1.27$ & $4.22 \pm 0.12$ \\
Sham + Y-26732 & $6.73 \pm 1.19^{\mathrm{a}}$ & $3.79 \pm 0.11^{\mathrm{a}}$ \\
Sham + Res & $8.67 \pm 1.00$ & $3.94 \pm 0.11$ \\
Sham + Res + U-46619 & $9.57 \pm 0.95$ & $3.76 \pm 0.51$ \\
Sham + Res + C3 & $8.06 \pm 1.50$ & $4.08 \pm 0.12$ \\
Sham + Res & $9.58 \pm 1.91$ & $3.98 \pm 0.22$ \\
Sham + Res + OA & $10.42 \pm 1.52$ & $4.01 \pm 0.06$ \\
Sham + Res + Y-26732 & $6.68 \pm 2.03^{\mathrm{b}}$ & $3.54 \pm 0.20^{\mathrm{b}}$ \\
LPS & $4.82 \pm 1.00$ & $3.92 \pm 0.29$ \\
LPS + U-46619 & $5.27 \pm 0.90$ & $3.90 \pm 0.17$ \\
LPS + U-46619 + Y-27632 & $3.75 \pm 0.92^{\mathrm{c}, \mathrm{d}}$ & $3.68 \pm 0.27$ \\
LPS & $5.09 \pm 1.24$ & $4.10 \pm 0.16$ \\
LPS + OA & $5.09 \pm 1.07$ & $4.19 \pm 0.18$ \\
LPS + OA + Y-27632 & $3.25 \pm 0.88^{\mathrm{c}, \mathrm{d}}$ & $3.56 \pm 0.21^{\mathrm{c}, \mathrm{d}}$ \\
LPS + Res & $8.38 \pm 0.61$ & $4.07 \pm 0.09$ \\
LPS + Res + C3 & $7.86 \pm 0.86$ & $4.01 \pm 0.17$ \\
LPS + Res + C3 + U-46619 & $9.20 \pm 1.26^{\mathrm{e}}$ & $3.88 \pm 0.23$ \\
LPS + Res & $8.25 \pm 0.70$ & $4.02 \pm 0.13$ \\
LPS + Res + Y-27632 & $4.93 \pm 0.68^{\mathrm{f}}$ & $3.63 \pm 0.11^{\mathrm{f}}$ \\
LPS + Res + Y-27632 + U-46619 & $6.38 \pm 0.93^{\mathrm{e}, \mathrm{f}}$ & $3.95 \pm 0.20^{\mathrm{e}}$ \\
& & \\
& & \\
& &
\end{tabular}

Data are presented as mean \pm standard error. ${ }^{a} \mathrm{P}<0.05$ vs. sham group; ${ }^{\mathrm{b}} \mathrm{P}<0.05$ vs. sham + Res group; ${ }^{\mathrm{C}} \mathrm{P}<0.05$ vs. LPS group; ${ }^{\mathrm{d}} \mathrm{P}<0.05$ vs. LPS + U46619 group or LPS + OA group; ${ }^{\mathrm{e}} \mathrm{P}<0.05$ vs. LPS + Res $+\mathrm{C} 3$ group or LPS + Res + Y-27632 group; ${ }^{\mathrm{P}} \mathrm{P}<0.05$ vs. LPS + Res group. Emax, maximum tension; $\mathrm{pD}_{2}$, negative log of molar concentration; Res, resveratrol; LPS, lipopolysaccharide; OA, okadaic acid.

$1 \times 10^{-5}, 3 \times 10^{-6}, 1 \times 10^{-6}, 3 \times 10^{-7}$ and $\left.1 \times 10^{-7} \mathrm{~mol} / \mathrm{l}\right)$, Emax and $\mathrm{PD}_{2}$ of arteriole rings harvested from mice of the LPS+Res group $(\mathrm{P}<0.05)$. RhoA agonist $\mathrm{U}-46619$ suppressed the effect of Y-27632 on vascular response at any concentration of NE, leading to significant increases at concentrations of $3 \times 10^{-7}$ and $1 \times 10^{-7} \mathrm{~mol} / 1$ and Emax, and significant decreases at concentrations of $3 \times 10^{-5}, 1 \times 10^{-5}$ and $3 \times 10^{-6} \mathrm{~mol} / 1$ when compared with that of the LPS + Res group $(\mathrm{P}<0.05)$. Furthermore, RhoA inhibitor C3 transferase exhibited an inhibiting trend on the response of arteriole rings harvested from mice of the LPS + Res group, but no significant statistical differences were observed. U-46619 plus C3 transferase co-incubation significantly increased the response and Emax of arteriole rings harvested from mice of the LPS + Res group compared with those incubated with $\mathrm{C} 3$ transferase $\left(3 \times 10^{-5}, 1 \times 10^{-5}, 3 \times 10^{-6}\right.$, $1 \times 10^{-6}, 3 \times 10^{-7}$ and $1 \times 10^{-7} \mathrm{~mol} / \mathrm{l}$ ) or not (at $3 \times 10^{-6}, 1 \times 10^{-6}, 3 \times 10^{-7}$ and $1 \times 10^{-7} \mathrm{~mol} / \mathrm{l} ; \mathrm{P}<0.05$; Fig. 4D).

Effect of Res treatment on the p-RhoA, p-Mypt1 and MLCP levels in vascular tissue of mice subjected to LPS challenge. 
A
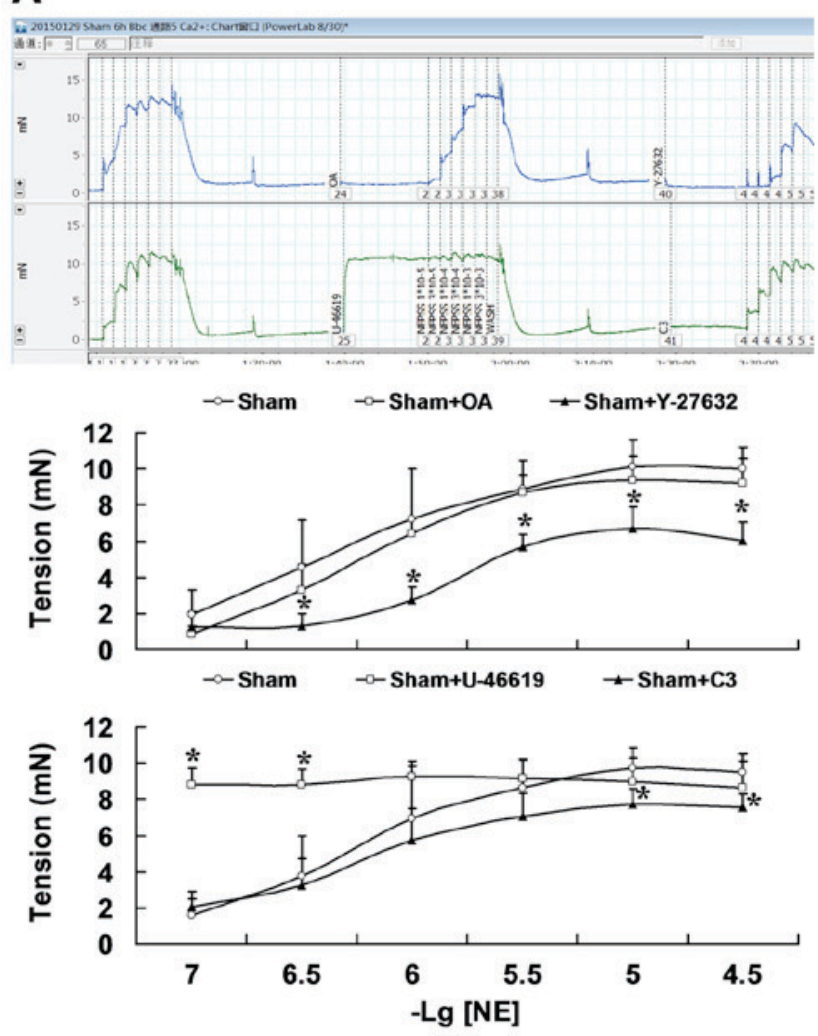

C
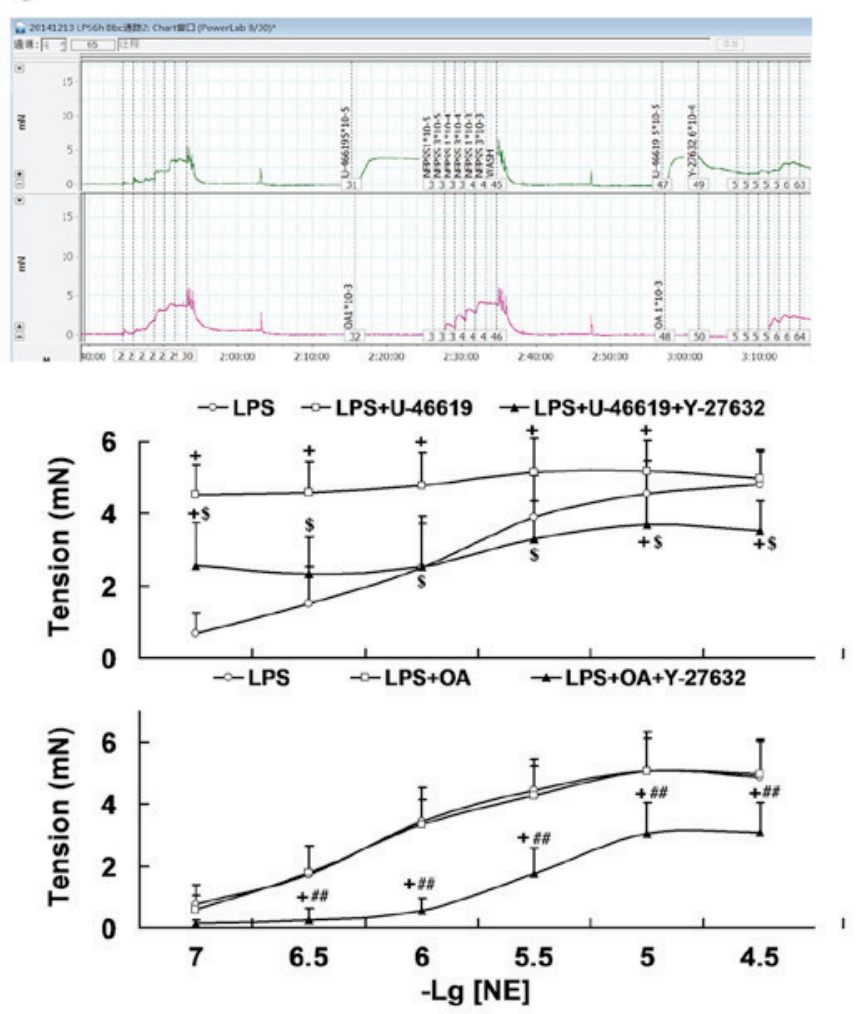

B
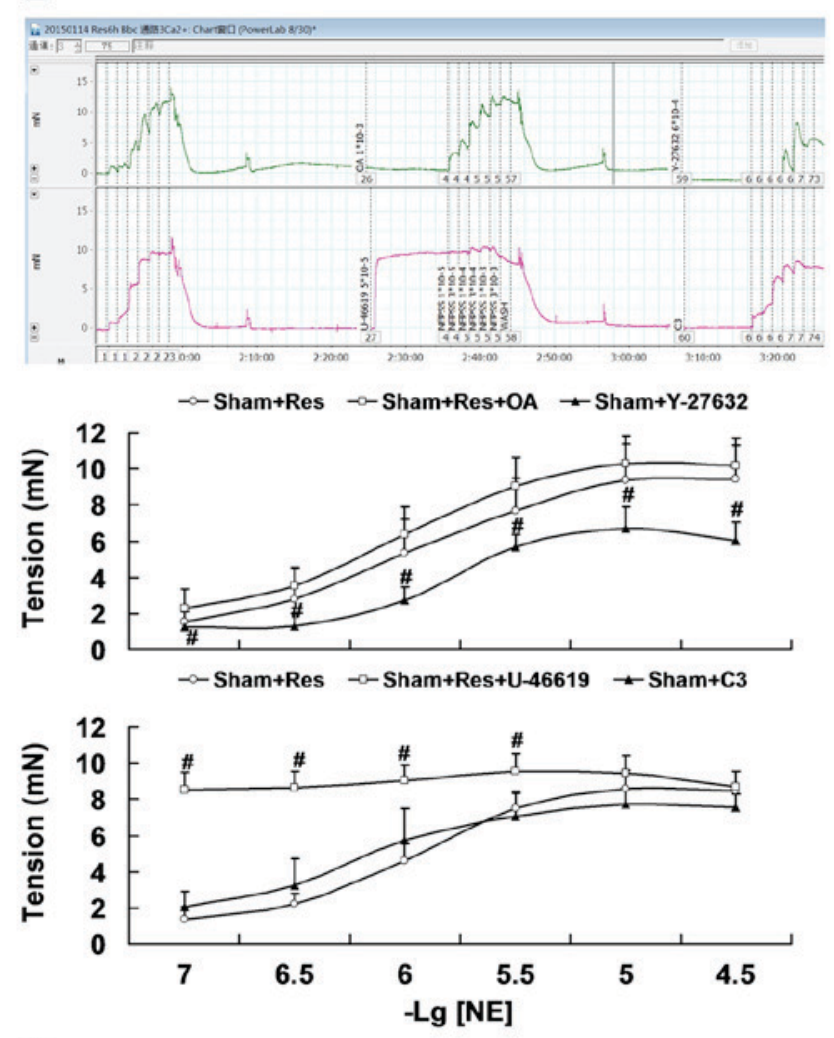

D
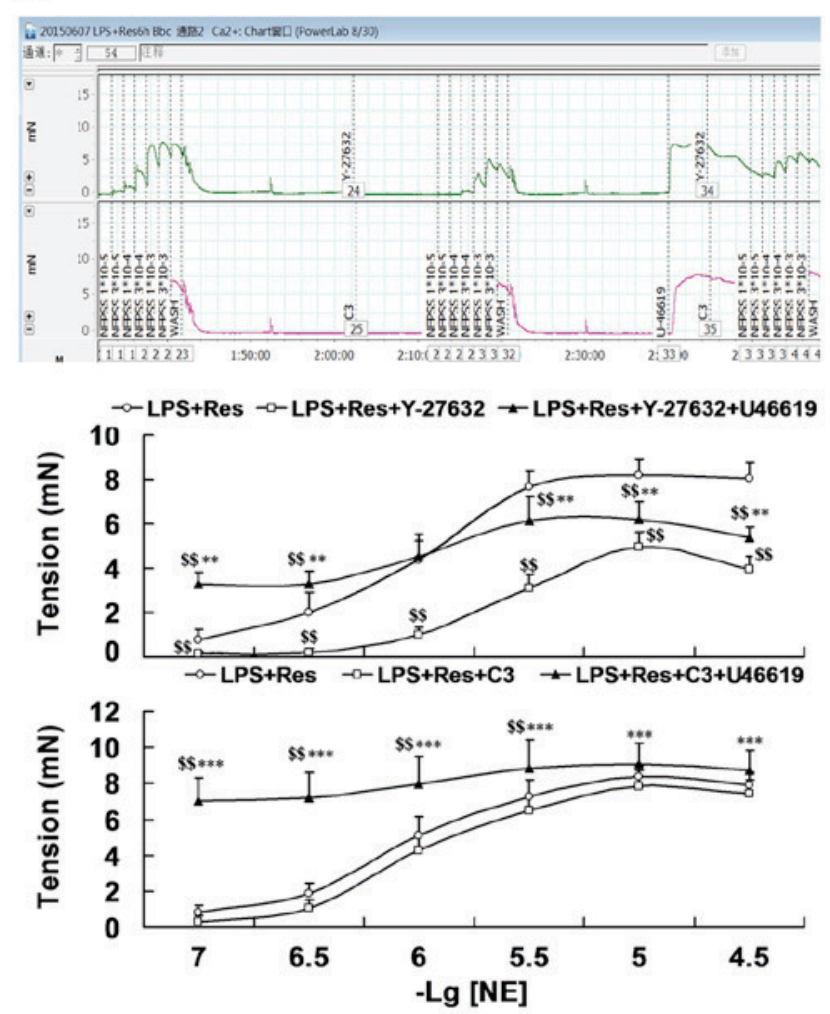

Figure 4. Role of agonists or inhibitors of the RhoA-Rho kinase-myosin light chain phosphatase pathway in the Res treatment enhancing micro-vascular reactivity to NE in mice following challenge with LPS. (A) Micro-vascular reactivity in the (A) sham group, (B) sham + Res group, (C) LPS group and (D) LPS + Res group. ${ }^{*} \mathrm{P}<0.05$ vs. the sham group; ${ }^{\#} \mathrm{P}<0.05$ vs. the sham + Res group; ${ }^{+} \mathrm{P}<0.05$ vs. the LPS group; ${ }^{\$} \mathrm{P}<0.05$ vs. $\mathrm{LPS}+\mathrm{U}-46619$ group; ${ }^{\# \#} \mathrm{P}<0.05$ vs. LPS + OA group; ${ }^{\$} \mathrm{P}<0.05$ vs. the LPS + Res group; ${ }^{* *} \mathrm{P}<0.05$ vs. LPS + Res + Y-27632 group; ${ }^{* * *} \mathrm{P}<0.05$ vs. LPS + Res + C3 group. Data are presented as mean \pm standard deviation, $n=6$. Res, resveratrol; NE, norepinephrine; LPS, lipopolysaccharide.

As presented in Fig. 5, the LPS challenge significantly decreased the p-RhoA and p-Mypt1 levels and significantly increased the MLCP level in vascular tissue $(\mathrm{P}<0.05)$, and these adverse effects were eradicated following treatment 
A

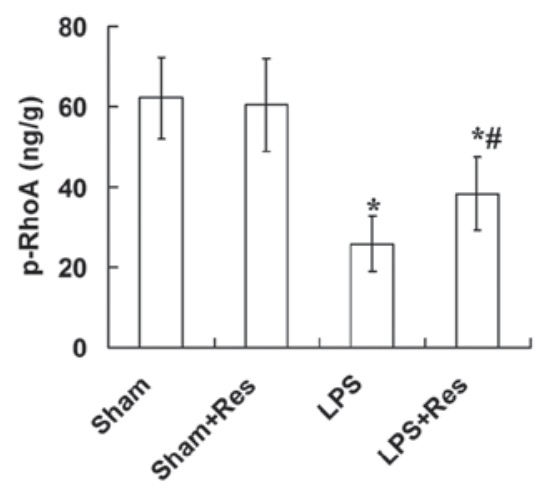

B

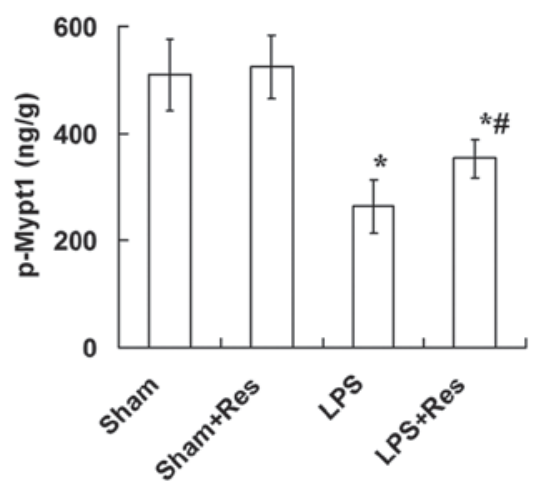

C



Figure 5. Effects of Res treatment on the levels of (A) p-RhoA, (B) p-Mypt1 and (C) MLCP in the mesenteric arteries of mice subject to lipopolysaccharide challenge. " $\mathrm{P}<0.05$ vs. sham and sham + Res groups; ${ }^{\sharp} \mathrm{P}<0.05$ vs. LPS group. Data are presented as mean \pm standard deviation, $\mathrm{n}=6$. Res, resveratrol; $\mathrm{p}-$, phosphorylated; MLCP, myosin light chain phosphatase; LPS, lipopolysaccharide.

with Res. However, the p-RhoA and p-Mypt1 levels in the LPS + Res group were significantly decreased compared with the sham and sham + Res groups $(\mathrm{P}<0.05)$.

\section{Discussion}

Previous breakthroughs indicate that endotoxemia is characterized by vascular hyporeactivity, which contributes to microcirculation failure and organ injury $(3,4)$. Therefore, vascular reactivity has been a focus of previous studies $(18,19)$; the present study demonstrated that post-treatment with Res significantly ameliorated LPS-induced vascular hyporeactivity, which was associated with the RhoA-ROCK-MLCP pathway. However, no marked effects of Res treatment on MAP and pressor response in LPS challenge mice were observed.

A number of methods have been used to establish endotoxic shock models, including the intravenous $(20-22)$ or intraperitoneal injection of LPS or gram-negative bacteria suspension (23-26) and cecal ligation and puncture (27-30), with each model representing the common clinical patient of endotoxic shock from different pathogenic factors. In the present study, the LPS challenge model was established through intravenous LPS injection, which reflects the shedding of LPS from gram-negative bacteria transposition into circulation, which results in endotoxemia. Based on the LPS challenge model, the present study investigated the effect of post-treatment of Res on survival time and revealed that Res treatment prolonged the survival time of mice. This result suggested that Res treatment inhibits the effect of the LPS challenge. The survival time and rate identified in the present study were similar to those indicated in previous research (31). In this previous study, intraperitoneal injection of highly purified LPS $(5 \mathrm{mg} / \mathrm{kg})$ resulted in a high mortality rate of $100 \%$ in wild-type C57 mice within $24 \mathrm{~h}$ post-injection. Because the mice in the LPS and LPS + Res groups were all sacrificed within $24 \mathrm{~h}$, the present study only compared the survival time between the LPS and LPS + Res groups, and did not observe the survival time of the Sham and Sham + Res group. The results of MAP indicated that LPS challenge induced a typical blood pressure curve of endotoxic shock, which briefly decreases in the early stage, then rises up before gradually declining. However, Res treatment was not observed to exhibit an evidently perfecting effect on MAP. A previous study reported that the ameliorating effect of post-treatment with Res on LPS-induced acute kidney injury was weaker when compared with Res pre-treatment (32). These previous findings suggested that the lack of a marked effect on MAP was associated with the opportunity of Res administration $1 \mathrm{~h}$ following LPS challenge. The results of the current study also demonstrated that the early application of Res treatment may be favorable for patients with sepsis.

Vascular reactivity is typically assessed through observing the pressor response of the whole animal to NE in vivo and the contractile response of artery rings to NE ex vivo (4). Thus, the current study measured the effect of Res on the pressor response of intravenous LPS-challenged mice and the contractile response to NE of microvessel rings from intravenous LPS-challenged mice. However, the results from in vivo and ex vivo experiments in the present study are inconsistent. The pressor response of mice to NE increased $6 \mathrm{~h}$ after the onset of intravenous infusion of LPS, whereas the contractile response to NE of isolated microvessel rings decreased. Res post-treatment increased the contractile response to NE of microvessel rings, but had no significant effect on the pressor response of mice.

Previous studies have demonstrated that an intravenous injection of LPS with a dose of $10 \mathrm{mg} / \mathrm{kg}$ reproduced vascular hyporeactivity to NE in vivo $(15,33)$, but no marked change was observed in the activity of NE following intraperitoneal injection of LPS with a dose of $8.5 \mathrm{mg} / \mathrm{kg}$ (34). Therefore, the present study hypothesized that the effect of LPS induced an increased pressor response to NE and may be associated with the lower dose of $5 \mathrm{mg} / \mathrm{kg}$ LPS. In addition, with the exception of the contractile apparatus and proteins in vascular smooth muscle cells (VSMCs), the pressor response in vivo is associated with a series of neuro-humoral factors, including a vasoconstrictive effect induced by sympathetic nervous excitement, catecholamines, angiotensin and vasopressin and a vasodilative effect of parasympathetic nervous hyper function, prostaglandins and nitric oxide (35). Thus, LPS injection may induce the sympathomimetic action by contributing to the vessel contraction and increase in pressor response. The detailed mechanism of LPS-induced increase in pressor response to NE requires further investigation. 
In general, the contractile tension of isolated vessels to vasoconstrictor substances is associated with the contractile apparatus and proteins in VSMCs, in addition to the function and content of adrenergic receptors (36-38). Therefore, LPS challenge-induced hyporeactivity of isolated vessels in the present study was caused by the decreased contractile function of VSMCs, which may be associated with the damage of contractile apparatus, the dysfunction of membrane ion channels and the desensitization of adrenal receptors. These results suggested that Res treatment improved the reactivity of isolated vessels to NE and was associated with the aforementioned targets. Furthermore, the present study also hypothesized that the discordance of pressor response and isolated vascular reactivity to NE may be associated with the weaker effect of LPS decreasing isolated vascular reactivity compared with sympathomimetic action. This may explain why the Res treatment did not affect the pressor response. Therefore, the mechanism by which Res treatment improved the isolated vascular reactivity of LPS-treated mice may be associated with the change of vasoconstrictor substances in mesenteric arteries or VSMCs.

Previous studies demonstrated that LPS-induced vascular hyporeactivity was associated with the suppression of small guanosine triphosphate-binding proteins, including RhoA, ROCK and non-muscular isoform of myosin light chain kinase activities $(15,39,40)$. However, the RhoA/ROCK pathway is involved in the hemorrhagic shock-induced vascular hyporeactivity (41-44). The current study observed the role of the RhoA/ROCK pathway in Res treatment enhancing vascular reactivity following LPS challenge, through the isolated vascular rings treated by tool reagents of RhoA, ROCK and MLCP ex vivo. The results of the present study indicated that the RhoA agonist and MLCP inhibitor eradicated the vascular hyporeactivity caused by challenge with LPS. The ROCK inhibitor may antagonize the effects of RhoA agonist and MLCP inhibitor, increasing vascular reactivity and the effect of ROCK inhibitor may be reversed by the RhoA agonist, U-46619. In addition, the effect of Res treatment enhancing vascular reactivity on LPS challenged mice was suppressed by ROCK inhibitor incubation, which was further eradicated by the RhoA agonist. Furthermore, RhoA inhibitor C3 transferase did not significantly inhibit the enhancing role of Res treatment, which was further increased by U-46619 plus C3 transferase co-incubation.

To assess the association between Res-treatment enhancing vascular reactivity and the RhoA-ROCK-MLCP signal pathway, the present study measured the effect of Res treatment on protein levels of p-RhoA, p-Mypt1 (which is a substrate of ROCK and reflects the activity of ROCK) and MLCP in the mesenteric arteries. The results of the present study indicated that after LPS treatment, Res treatment increased the level of RhoA and p-Mypt1 and decreased the level of MLCP. Combined with the results of the ex vivo experiment; while the inhibiting effect of the ROCK inhibitor Y-27632 on Res treatment was suppressed by the RhoA agonist U-46619 treatment and the increasing effect of U-46619 on LPS-induced vascular hyporeactivity were eradicated by Y-27632 incubation, the present findings suggested that the mechanism by which LPS challenge was eliciting vascular hyporeactivity is associated with the suppression of the RhoA-ROCK-MLCP signal pathway. Furthermore, the vascular protective effect of Res treatment for LPS challenge is also associated with this signal pathway.
A number of studies have indicated that the RhoA-ROCK pathway has various pathophysiologic roles in the process of cell death or apoptosis (45), reactive oxygen species generation (46), cytoskeleton remodeling (47) and vasoconstriction $(15,42)$. However, the mechanism of activation or suppression for the RhoA-ROCK pathway remains unknown. The detailed mechanism by which Res activates the RhoA-ROCK-MLCP pathway following LPS challenge also requires further research.

In addition, Liang et al (4) reported that the suppression of vascular $\alpha 1$ adrenergic receptors expression was involved in LPS-induced vascular hyporeactivity. Therefore, whether or not Res treatment may enhance vascular $\alpha 1$ adrenergic receptors expression requires further investigation. A number of studies have provided evidence that mitochondrial dysfunction is involved in hemorrhagic shock-induced vascular hyporeactivity $(48,49)$. Res treatment may attenuate the mitochondrial dysfunction of VSMCs induced by serious hemorrhagic shock, enhance ATP levels, reduce the content of lipid peroxides and improve the vascular reactivity of hemorrhagic shock animals $(50,51)$. Therefore, whether the LPS challenge elicited mitochondrial injury of VSMCs and whether Res improves vascular reactivity may be associated with its mitochondrial protective effect remains to be elucidated.

In conclusion, the present findings demonstrated that Res attenuates the vascular hyporeactivity and survival time of LPS challenged mice, but no significant effects were observed regarding MAP and pressor response for the whole animal. The inconsistent results of pressor response in vivo and contractile response of isolated vascular rings to NE may be associated with the LPS-induced sympathomimetic effect, but the mechanism requires further investigation. The present study presents a novel underlying role of Res on LPS-induced sepsis and further investigation of the mechanism by which Res elevates vascular reactivity may provide novel insights for the associated diseases in clinical practice.

\section{References}

1. Maloney PJ: Sepsis and septic shock. Emerg Med Clin North Am 31: 583-600, 2013.

2. Angus DC and van der Poll T: Severe sepsis and septic shock. N Engl J Med 369: 840-851, 2013.

3. Tsao CM, Chen SJ, Tsou MY and Wu CC: Effect of propofol on vascular reactivity in thoracic aortas from rats with endotoxemia. J Chin Med Assoc 75: 262-268, 2012.

4. Liang JL, Yang GM, Li T and Liu LM: Interleukin $1 \beta$ attenuates vascular $\alpha 1$ adrenergic receptors expression following lipopolysaccharide-induced endotoxemia in rabbits: Involvement of JAK2-STAT3 pathway. J Trauma Acute Care Surg 76: 762-770, 2014.

5. Piotrowska H, Kucinska M and Murias M: Biological activity of piceatannol: Leaving the shadow of resveratrol. Mutat Res 750: 60-82, 2012.

6. KuršvietienėL, Stanevičienè I, Mongirdienė A and Bernatonienė J: Multiplicity of effects and health benefits of resveratrol. Medicina (Kaunas) 52: 148-155, 2016.

7. Kolgazi M, Sener G, Cetinel S, Gedik N and Alican I: Resveratrol reduces renal and lung injury caused by sepsis in rats. J Surg Res 134: 315-321, 2006.

8. Xu W, Lu Y, Yao J, Li Z, Chen Z, Wang G, Jing H, Zhang X, Li M, Peng J and Tian X: Novel role of resveratrol: Suppression of high-mobility group protein box 1 nucleocytoplasmic translocation by the upregulation of sirtuin 1 in sepsis-induced liver injury. Shock 42: 440-447, 2014.

9. Zhang HX, Duan GL, Wang CN, Zhang YQ, Zhu XY and Liu YJ: Protective effect of resveratrol against endotoxemia-induced lung injury involves the reduction of oxidative/nitrative stress. Pulm Pharmacol Ther 27: 150-155, 2014. 
10. Sebai H, Sani M, Aouani E and Ghanem-Boughanmi N: Cardioprotective effect of resveratrol onlipopolysaccharide-induced oxidative stress in rat. Drug Chem Toxicol 34: 146-150, 2011.

11. Sebai H, Sani M, Yacoubi MT, Aouani E, Ghanem-Boughanmi N and Ben-Attia M: Resveratrol, a red wine polyphenol, attenuates lipopolysaccharide-induced oxidative stress in rat liver. Ecotoxicol Environ Saf 73: 1078-1083, 2010.

12. Cao Q, Jing C, Tang X, Yin Y, Han X and Wu W: Protective effect of resveratrol on acute lung injury induced by lipopolysaccharide in mice. Anat Rec (Hoboken) 294: 527-532, 2011.

13. Holthoff JH, Wang Z, Seely KA, Gokden N and Mayeux PR: Resveratrol improves renal microcirculation, protects the tubular epithelium, and prolongs survival in a mouse model of sepsis-induced acute kidney injury. Kidney Int 81: 370-378, 2012.

14. Tseng TL, Chen MF, Liu CH, Pang CY, Hsu YH and Lee TJ: Induction of endothelium-dependent constriction of mesenteric arteries in endotoxemic hypotensive shock. Br J Pharmacol 173: 1179-1195, 2016.

15. Liao MH, Shih CC, Tsao CM, Chen SJ and Wu CC: RhoA/Rho-kinase and nitric oxide in vascular reactivity in rats with endotoxaemia. PLoS One 8: e56331, 2013.

16. Smeding L, Leong-Poi H, Hu P, Shan Y, Haitsma JJ, Horvath E, Furmli S, Masoom H, Kuiper JW, Slutsky AS, et al: Salutary effect of resveratrol on sepsis-induced myocardial depression. Crit Care Med 40: 1896-1907, 2012.

17. Lai X, Pei Q, Song X, Zhou X, Yin Z, Jia R, Zou Y, Li L, Yue G, Liang $\mathrm{X}$, et al: The enhancement of immune function and activation of NF- $\kappa$ B by resveratrol-treatment in immunosuppressive mice. Int Immunopharmacol 33: 42-47, 2016.

18. Liu LM, Ward JA and Dubick MA: Hemorrhage-induced vascular hyporeactivity to norepinephrine in select vasculatures of rats and the roles of nitric oxide and endothelin. Shock 19: 208-214, 2003.

19. Zhao ZG, Niu CY, Wei YL, Zhang YP, Si YH and Zhang J: Mesenteric lymph return is an important contributor to vascular hyporeactivity and calcium desensitization after hemorrhagic shock. Shock 38: 186-195, 2012.

20. Li A, Dong L, Duan ML, Sun K, Liu YY, Wang MX, Deng JN, Fan JY, Wang BE and Han JY: Emodin improves lipopolysaccharide-induced microcirculatory disturbance in rat mesentery. Microcirculation 20: 617-628, 2013

21. Zhang Y, Sun K, Liu YY, Zhang YP, Hu BH, Chang X, Yan L, Pan CS, Li Q, Fan JY, et al: Ginsenoside Rb1 ameliorates lipopolysaccharide-induced albumin leakage from rat mesenteric venules by intervening in both trans- and paracellular pathway. Am J Physiol Gastrointest Liver Physiol 306: G289-G300, 2014.

22. Pei H, Zhang Y, Wu H, Ren L, Jia X, Zhang Y and Chen M: Relationship between iNOS expression and apoptosis in cerebral tissue, and the effect of sini injection in endotoxin shock rats. J Tradit Chin Med 33: 486-491, 2013.

23. Li Y, Liu B, Fukudome EY, Lu J, Chong W, Jin G, Liu Z, Velmahos GC, Demoya M, King DR and Alam HB: Identification of citrullinated histone $\mathrm{H} 3$ as a potential serum protein biomarker in a lethal model of lipopolysaccharide-induced shock. Surgery 150: 442-451, 2011.

24. Liu C, Zhang GF, Song SW, Cai GJ, Liu WH, Miao CY and Su DE: Effects of ketanserin on endotoxic shock and baroreflex function in rodents. J Infect Dis 204: 1605-1612, 2011.

25. Madonna R, Jiang J and Geng YJ: Attenuated expression of gelsolin in association with induction of aquaporin-1 and nitric oxide synthase in dysfunctional hearts of aging mice exposed to endotoxin. Int J Immunopathol Pharmacol 25: 911-922, 2012.

26. Noworyta-Sokołowska K, Górska A and Gołembiowska K LPS-induced oxidative stress and inflammatory reaction in the rat striatum. Pharmacol Rep 65: 863-869, 2013

27. Park EJ, Jang HJ, Tsoyi K, Kim YM, Park SW, Kim HJ, Lee JH and Chang KC: The heme oxygenase-1 inducer THI-56 negatively regulates iNOS expression and HMGB1 release in LPS-activated RAW 264.7 cells and CLP-induced septic mice. PLoS One 8: e76293, 2013

28. Zhao T, Li Y, Liu B, Liu Z, Chong W, Duan X, Deperalta DK, Velmahos GC and Alam HB: Novel pharmacologic treatment attenuates septic shock and improves long-term survival. Surgery 154: 206-213, 2013.

29. Kang K, Nan C, Fei D, Meng X, Liu W, Zhang W, Jiang L, Zhao M, Pan S and Zhao M: Heme oxygenase 1 modulates thrombomodulin and endothelial protein $\mathrm{C}$ receptor levels to attenuate septic kidney injury. Shock 40: 136-143, 2013.

30. Olguner CG, Koca U, Altekin E, Ergür BU, Duru S, Girgin P, Taşdöğen A, Gündüz K, Güzeldağ S, Akkus M and Micili SC: Ischemic preconditioning attenuates lipid peroxidation and apoptosis in the cecal ligation and puncture model of sepsis. Exp Ther Med 5: 1581-1588, 2013.
31. Buchholz BM, Chanthaphavong RS, Billiar TR and Bauer AJ: Role of interleukin- 6 in hemopoietic and non-hemopoietic synergy mediating TLR4-triggered late murine ileus and endotoxic shock. Neurogastroenterol Motil 24: 658-669, e294, 2012.

32. Chen L, Yang S, Zumbrun EE, Guan H, Nagarkatti PS and Nagarkatti M: Resveratrol attenuates lipopolysaccharide-induced acute kidney injury by suppressing inflammation driven by macrophages. Mol Nutr Food Res 59: 853-864, 2015.

33. Fatehi-Hassanabad Z, Muller B, Andriantsitohaina R, Furman BL, Parratt JR and Stoclet JC: Influence of indomethacin on the haemodynamic effects of lipopolysaccharide in rats. Fundam Clin Pharmacol 10: 258-263, 1996

34. Boffa JJ and Arendshorst WJ: Maintenance of renal vascular reactivity contributes to acute renal failure during endotoxemic shock. J Am Soc Nephrol 16: 117-124, 2005.

35. Shaikh WA, Patel M, Shah HD, Nimbalkar AS, Patel N and Singh SK: Arterial blood pressure is inversely associated with vascular sympathetic reactivity (isometric handgrip exercise) in Gujarati Indian adolescents. Indian J Physiol Pharmacol 58: 271-274, 2014.

36. Bing RJ, Termin A, Conforto A, Dudek R and Hoffmann MJ: Membrane function and vascular reactivity. Biosci Rep 13: 61-67, 1993.

37. Ponnoth DS and Jamal Mustafa S: Adenosine receptors and vascular inflammation. Biochim Biophys Acta 1808: 1429-1434, 2011.

38. Guzmán-Gutiérrez E, Arroyo P, Salsoso R, Fuenzalida B, Sáez T, Leiva A, Pardo F and Sobrevia L: Role of insulin and adenosine in the human placenta microvascular and macrovascular endothelial cell dysfunction in gestational diabetes mellitus. Microcirculation 21: 26-37, 2014.

39. Liang JL, Yang GM, Li T and Liu LM: Effects of interleukin-1ß on vascular reactivity after lipopolysaccharide-induced endotoxic shock in rabbits and its relationship with PKC and Rho kinase. J Cardiovasc Pharmacol 62: 84-89, 2013.

40. Recoquillon S, Carusio N, Lagrue-Lakhal AH, Tual-Chalot S, Filippelli A, Andriantsitohaina R and Martinez MC: Interaction in endothelium of non-muscular myosin light-chain kinase and the NF- $\mathrm{KB}$ pathway is critical to lipopolysaccharide-induced vascular hyporeactivity. Clin Sci (Lond) 129: 687-698, 2015.

41. Hu Y, Yang G, Xiao X, Liu L and Li T: Bkca opener, NS1619 pretreatment protects against shock-induced vascular hyporeactivity through PDZ-Rho GEF-RhoA-Rho kinase pathway in rats. J Trauma Acute Care Surg 76: 394-401, 2014.

42. Zhao Z, Si Y, Zhang Y, Du S, Zhang L and Niu C: Postshock mesenteric lymph drainage ameliorates vascular reactivity and calcium sensitivity through RhoA. J Surg Res 186: 304-309, 2014.

43. Li T, Fang Y, Yang G, Xu J, Zhu Y and Liu L: Effects of the balance in activity of RhoA and Racl on the shock-induced biphasic change of vascular reactivity in rats. Ann Surg 253: 185-193, 2011.

44. Hu Y, Li T, Tang XF, Chen K and Liu L: Effects of ischemic preconditioning on vascular reactivity and calcium sensitivity after hemorrhagic shock and their relationship to the RhoA-Rho-kinase pathway in rats. J Cardiovasc Pharmacol 57: 231-239, 2011.

45. Okumura N, Fujii K, Kagami T, Makiko N, Kitahara M, Kinoshita S and Koizumi N: Activation of the Rho/Rho kinase signaling pathway is involved in cell death of corneal endothelium. Invest Ophthalmol Vis Sci 57: 6843-6851, 2016.

46. Akbar H, Duan X, Saleem S, Davis AK and Zheng Y: RhoA and Rac1 GTPases differentially regulate agonist-receptor mediated reactive oxygen species generation in platelets. PLoS One 11: e0163227, 2016.

47. Bang J, Jang M, Huh JH, Na JW, Shim M, Carlson BA, Tobe R, Tsuji PA, Gladyshev VN, Hatfield DL and Lee BJ: Deficiency of the $15-\mathrm{kDa}$ selenoprotein led to cytoskeleton remodeling and non-apoptotic membrane blebbing through a RhoA/ROCK pathway. Biochem Biophys Res Commun 456: 884-890, 2015.

48. Lei Y, Peng X, Liu L, Dong Z and Li T: Beneficial effect of cyclosporine A on traumatic hemorrhagic shock. J Surg Res 195: 529-540, 2015.

49. Fang Y, Li T, Fan X, Zhu Y and Liu L: Beneficial effects of activation of PKC on hemorrhagic shock in rats. J Trauma 68: 865-873, 2010

50. Wang X, Song R, Bian HN, Brunk UT, Zhao M and Zhao KS: Polydatin, a natural polyphenol, protects arterial smooth muscle cells against mitochondrial dysfunction and lysosomal destabilization following hemorrhagic shock. Am J Physiol Regul Integr Comp Physiol 302: R805-R814, 2012.

51. Wang X, Song R, Chen Y, Zhao M and Zhao KS: Polydatin - a new mitochondria protector for acute severe hemorrhagic shock treatment. Expert Opin Investig Drugs 22: 169-179, 2013. 